\title{
In vivo Assessment of Cold Atmospheric Pressure Plasma Technology on the Bioactivity of Spirulina
}

\author{
María Consuelo Pina-Pérez ${ }^{1,2}$, María Úbeda-Manzanaro ${ }^{3}$, Michael Beyrer $^{2}$, \\ Antonio Martínez ${ }^{3}$ and Dolores Rodrigo ${ }^{3 *}$ \\ 'Departamento de Microbiología y Ecología, Universitat de València, Burjassot, Valencia, Spain, ${ }^{2}$ Food Engineering \\ Laboratory, Institute of Life Technologies, University of Applied Sciences and Arts Western-Switzerland (HES-SO) \\ Valais-Wallis, Sion, Switzerland, ${ }^{3}$ Departamento Conservación y Calidad, Instituto de Agroquímica y Tecnología \\ de Alimentos IATA - Consejo Superior de Investigaciones Científicas (CSIC), Valencia, Spain
}

OPEN ACCESS

Edited by:

Panagiotis Svarnas,

University of Patras, Greece

Reviewed by:

Veronica Ortiz Alvarenga,

Federal University of Minas Gerais,

Brazil

Piotr Kulawik, University of Agriculture in Krakow,

Poland

*Correspondence:

Dolores Rodrigo

lolesra@iata.csic.es

Specialty section: This article was submitted to Antimicrobials, Resistance and Chemotherapy,

a section of the journal

Frontiers in Microbiology

Received: 23 September 2021 Accepted: 24 December 2021 Published: 24 January 2022

Citation: Pina-Pérez MC, Úbeda-Manzanaro M, Beyrer M, Martínez A and Rodrigo D (2022)

In vivo Assessment of Cold Atmospheric Pressure Plasma Technology on the Bioactivity of

Spirulina.

Front. Microbiol. 12:781871. doi: 10.3389/fmicb.2021.781871
The present study challenges the in vivo assessment of cold atmospheric pressure plasma (CAPP) technology on the bioactive activity (antioxidant/antiaging and antimicrobial potential) of Spirulina powder, using Caenorhabditis elegans as an animal model. Surface microdischarge cold atmospheric pressure plasma (SMD-CAPP) treatment was $3.3 \mathrm{~W}$ discharge power for $7 \mathrm{~min}$. C. elegans lifespan and egg laying were used as indicators of antioxidant/antiaging potential of Spirulina (1 mg/mL), when grown with Spirulina CP-treated [E_SCP] and untreated [E_S], compared with a control [E_O] (non-supplemented with Spirulina). According to our results, under both Spirulina supplemented media [E_SCP and E_S] and for the first 17 days, nematodes experienced an increase in lifespan but without significant differences $(p>0.05)$ between control and Spirulina CP-treated. Regarding the in vivo assay of the antimicrobial potential of Spirulina against Salmonella enterica serovar Typhimurium (infected worms), no significant differences $(p>0.05)$ were found between the three exposure scenarios (control [S_0]; Spirulina supplemented media [S_S]; CP-treated Spirulina supplemented media [S_SCP]). According to present results, CAPP-treatment do not influence negatively the lifespan of $C$. elegans but a reduction in the Spirulina antiaging potential was found. No in vivo modifications in antimicrobial activity seem to be linked to CAPP-processed Spirulina.

Keywords: Spirulina (Arthospira) platensis, cold atmospheric plasma (CAP), in vivo studies, C. elegans, antimicrobial activity, antioxidant activity

\section{INTRODUCTION}

In recent years, there has been a steady growth of scientific interest in blue green microalgae Arthospira platensis, hereafter referred to as Spirulina, as a sustainable source of proteins and other high-value compounds with physiological properties (Ozdemir et al., 2004; Khan et al., 2005, 2006; Nuhu, 2013). Spirulina platensis, and its purified extracts, have demonstrated functional properties, of which the most relevant include potential antioxidant, antimicrobial, anti-cancer, and immunomodulatory properties (El-Baky et al., 2008; Nuhu, 2013; Marco Castro et al., 2019). 
Specifically, the antimicrobial potential of Spirulina has been attributed to intracellular and extracellular synthetized metabolites, such as terpenols, sterols, polysaccharides, dibutenolides, peptides, and proteins, secreted by or present in Spirulina with demonstrated bactericidal and bacteriostatic effects against clinical and foodborne human pathogens (Lee et al., 2014; Pina-Pérez et al., 2017; Seghiri et al., 2019). The antimicrobial capacity of these Spirulina bioactive molecules is currently being exploited in useful applications by the food industry, and pharmacological and cosmetic sectors (Grahl et al., 2018; Martelli et al., 2020).

One of the most pertinent aspects of novel Spirulina-derived product formulation is related with the stability/functionality maintenance of bioactive molecules after processing (proteins, peptides, lipids, polyphenols, polysaccharides) (Chaiklahan et al., 2012; Pina-Pérez et al., 2018; Cassani et al., 2020). Recent trends are moving toward more efficient, environmentally friendly, and very rapid non-thermal treatments (ranging from a few seconds to a few minutes), implemented in the food and pharmaceutical industries to inactivate pathogenic bacteria, while preserving the techno-functional product properties intact (flavor, color, texture, solubility) as well as the beneficial bioactive potential in the end product. Among these novel processes, cold plasma (CP) is emerging as a non-thermal technology, with huge versatility to treat solid, liquid and powder-based matrices, proving effective in the inactivation of spores, viruses, mycotoxins and prions (Elmoualij et al., 2012; ten Bosch et al., 2017; Beyrer et al., 2020; Pina-Pérez et al., 2020). Plasma has been defined as the fourth state of matter, a partially ionized gas (Helium, Argon, air, other mixtures) with unique properties. Radio-frequency, microwaves, thermal energy, electric and magnetic fields [plasma jet, surface microdischarge plasma (SMD), dielectric barrier discharge plasma (DBD)] are used as energy sources for gas ignition, with the generated plasma being comprised by ions (positive and negative), free electrons, radicals, and electromagnetic radiation (photons UV and visible light) (Tolouie et al., 2018). To date, the continuous generation of electrical microdischarges is the most efficient method of choice to generate cold plasma (temperature $30-40^{\circ} \mathrm{C}$ ) (Pina-Pérez et al., 2020).

In spite of the promising results obtained so far in terms of the microbiological safety obtained by CP application, some effects still remain unknown (Whitehead, 2016; Ziuzina and Misra, 2016). In fact, the complex chemistry generated by plasma ignition (hydroxyl radicals $(\mathrm{OH})$, hydrogen peroxide $\left(\mathrm{H}_{2} \mathrm{O}_{2}\right)$, ozone $\left(\mathrm{O}_{3}\right)$, superoxide anion radicals $\left(\bullet \mathrm{O}^{2-}\right)$, atomic oxygen $(\mathrm{O})$, nitric oxide $(\mathrm{NO})$, nitrite/nitrate $\left(\mathrm{NO}_{2}{ }^{-} / \mathrm{NO}_{3}{ }^{-}\right)$, and subsequently, the dynamic interaction of plasma reactive species with food macromolecules requires [food-plasma treatment] in-depth evaluation. These mainly concern the bioactive structure (side-toxic compounds) and functionality (antioxidant or antimicrobial activity, among others) after processing (Sarangapani et al., 2017; Pérez-Andrés et al., 2019). To date, very few studies (mainly focused on lipids oxidation and proteins denaturation) have been published regarding potential biological risks associated to cold plasma when used in food/pharmaceutical processing (for instance regarding the functional effect of these novel CP processed matrices) (Gavahian et al., 2018; Alves Filho et al., 2019).

In spite of the rich-nutritional value of the Spirulina matrix, including complex polysaccharides, vitamins and polyphenols with antiaging properties (scavenging free radicals, reducing DNA damage, and inhibiting ROS accumulation) and antimicrobial capacity, no previous studies have evaluated Spirulina functionality in vivo after CAPP processing. Therefore, the present study aims to evaluate the effect of cold atmospheric pressure plasma technology on the antioxidant/antiaging and antimicrobial bioactive potential of Spirulina against Salmonella enterica serovar Typhimurium, by using Caenorhabditis elegans as in vivo model.

\section{MATERIALS AND METHODS}

\section{Spirulina Powder}

A Spirulina (Arthrospira platensis) powder (Spirulina Plus) was purchased from Phytopharma S.A. (Grandvillard, Switzerland).

\section{Surface Microdischarge Cold Atmospheric Pressure Plasma Treatment}

In the present study, a Surface Microdischarge Cold Atmospheric Pressure Plasma (SMD-CAPP) equipment was used fully developed and constructed by the Institute of Systems Engineering (HES-SO Valais-Wallis, Sion, Switzerland). The system is built basically with a high-voltage powered planar, stainless steel grid electrode (total surface area $=149.76 \mathrm{~cm}^{2}$; grid size $=9.8 \times 9.4 \mathrm{~mm}^{2}$ ), mounted with a dielectric barrier made from Teflon, and a water-cooled ground electrode. Plasma is ignited on air. The distance between the plasma active zone (powered electrode) and the sample is $6 \mathrm{~mm}$. The electric circuit was described before (Pina-Pérez et al., 2020). The CAPP treatment settings were selected according to the demonstrated $\geq 2 \log 10$ inactivation of $B$. subtilis spores embedded in a starch powder (Beyrer et al., 2020). In short, a discharge power of $3.3 \mathrm{~W}$ was applied on a thin layer of Spirulina powder homogeneously spread on sterile glass slides $\left(0.5 \mathrm{mg} / \mathrm{cm}^{2}\right)$. The treatment time was $7 \mathrm{~min}$ and the voltage frequency $10 \mathrm{kHz}$.

\section{Caenorhabditis elegans Studies}

In the present study, the nematode C. elegans strain $\mathrm{N} 2$ was used, provided by the College of Biological Sciences, Minnesota University, United States. Nematodes were routinely maintained in NGM (Nematode Growth Media) (Stiernagle, 2006) petri dishes, in a bacterial lawn of E. coli OP50 (noninfected studies) or Salmonella Typhimurium (CECT 443) (infected studies) (Table 1). The worms at larval stage L4 were obtained by synchronization (Sanz-Puig et al., 2020). For lifespan studies, once synchronized, L4 nematodes (initially 250), were periodically transferred to plates (25 plates; 10 nematodes per plate), maintained at $20^{\circ} \mathrm{C}$ during their life cycle (approximately 3 weeks), and examined at $48 \mathrm{~h}$ intervals with a binocular microscope (COMECTA S.A.). Worms were considered dead 
TABLE 1 | Groups studied and feeding media.

\begin{tabular}{lll}
\hline & Group & Feeding media $^{\mathbf{a}}$ \\
\hline Non-infected & Control [E_0] & E. coli OP50 \\
& Spirulina [E_S] & $\begin{array}{l}\text { E. coli OP50 }+1 \mathrm{mg} / \mathrm{mL} \\
\text { non-treated Spirulina }\end{array}$ \\
& Spirulina CP-treated [E_SCP] & $\begin{array}{l}\text { E. coli OP50 }+1 \mathrm{mg} / \mathrm{mL} \\
\text { CP-treated Spirulina }\end{array}$ \\
Infected & Control [S_0] & S. Typhimurium \\
& Spirulina [S_S] & S. Typhimurium $+1 \mathrm{mg} / \mathrm{mL}$ \\
& Spirulina CP-treated [S_SCP] & S. Typhimurium $+1 \mathrm{mg} / \mathrm{mL}$ \\
& & CP-Treated Spirulina
\end{tabular}

a Nematodes belonging to all groups were maintain in NGM agar with the addition of the different microorganism cultures and Spirulina, depending on the study group.

when they did not move or do not respond to stimulation (contact with a platinum worm picker).

For nematode egg laying studies, 25 adult worms were distributed in 25 plates per substrate under study. Plates were incubated at $20^{\circ} \mathrm{C}$ for 48 or $72 \mathrm{~h}$. After this time, the progeny (eggs and larvae) of each adult worm was counted and the worm was transferred to a new plate of the same substrate. The procedure was repeating until the nematode's death.

Caenorhabditis elegans lifespan and egg-laying capacity were recorded for the different study and control groups (Table 1). For non-infected assays, NGM plates with non-CP treated Spirulina [E_S] and CP-treated Spirulina, [E_SCP], were seeded with a bacterial lawn of E. coli OP50. For infected studies, plates with non-CP treated Spirulina [S_S] and CP-treated Spirulina [S_CPS] were seeded with a $S$. Typhimurium bacterial lawn to simulate infection Mathematical modeling and statistical analysis.

The Weibull distribution function was fit to the survival curves (Mafart et al., 2002) (Equation 1). To do so, Ginafit software was used (Geeraerd et al., 2005).

$$
\log _{10}(N)=\log _{10}\left(\left(\frac{t}{\delta}\right)^{p}\right)
$$

where $\mathrm{N}$ is the number of alive worms at time $t, \mathrm{~N}_{0}$ is the number of worm population at time zero $\left(\mathrm{t}_{0}\right), \partial$ is the kinetic parameter (days/worms) and represents the time of first decimal reduction for a specific worm and, $\mathrm{p}$ is the shape parameter of the Weibull distribution function.

Kaplan-Meirer estimator (Kaplan and Meier, 1958) was also used to fit survival experimental data as a function of time.

$$
S(t)=\prod_{t_{i} \leq t}\left(1-\frac{d_{i}}{n_{i}}\right)
$$

Where, $\mathrm{t}_{i}$ is a time when at least one event happened, $\mathrm{d}_{i}$ is the number of events that happened at time $t_{i}$, and $n_{i}$ represents the number of individuals known to have survived up to time $t_{i}$.

Percentiles for survival curves were obtained for each experimental group, and ANOVA analysis were performed to determine significant differences between groups. Statgraphics Centurion XVII software was used for these analyses.

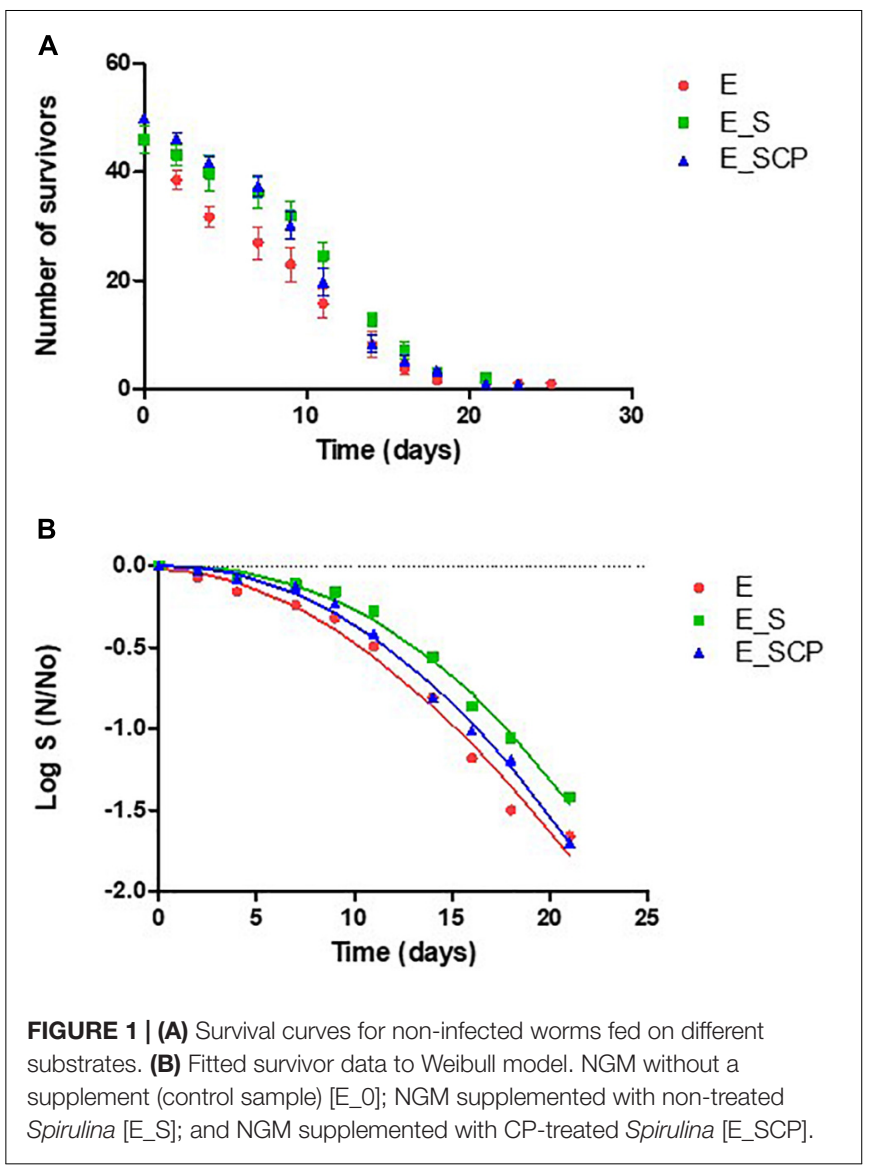

\section{RESULTS}

\section{Effect of Cold Atmospheric Pressure Plasma Treatment of Spirulina on the Lifespan and Reproductive-Rate of Caenorhabditis elegans Populations}

The lifespan assay is an index used to evaluate the bioactive potential (antiaging effect) of different compounds (Ayyadevara et al., 2013; Jattujan et al., 2018; Chen et al., 2020; IbáñezPeinado et al., 2020). The cumulative survival curves of the nematode population fed with the different substrates can be seen in Figure 1. It clearly shows that the number of live worms decreased over time until approximately 21 days for all populations studied. In the case of the nematode population exposed to Spirulina [E_S] and [E_SCP], lower nematode death rates per time interval compared to control samples appear, probably due to a protective antiaging effect due to Spirulina exposure, regardless of whether the Spirulina had been treated with cold plasma [E_SCP] or not [E_S].

Survival and censored data for each time interval were analyzed with the Kaplan Meier estimator in order to obtain the percentile tables indicating the number of nematodes that survive up to a certain time point (Table 2). Significant differences on the lifespan of the control and nematodes fed with untreated Spirulina supplement $(p \leq 0.05)$ were determined for all 
TABLE 2 | Lifespan (days) of non-infected nematodes for the different percentile depending on group studied.

\begin{tabular}{lccc}
\hline $\begin{array}{l}\text { Percentile of } \\
\text { survivors [\%] }\end{array}$ & \multicolumn{3}{c}{ Lifespan of nematodes [d] } \\
\cline { 2 - 4 } & $\begin{array}{c}\text { Control } \\
\text { [E_0] }\end{array}$ & $\begin{array}{c}\text { Untreated } \\
\text { Spirulina } \\
\text { [E_S] }\end{array}$ & $\begin{array}{c}\text { CP treated } \\
\text { Spirulina } \\
\text { [E_SCP] }\end{array}$ \\
\hline 75 & $5.20 \pm 0.42^{\mathrm{a}}$ & $7.83 \pm 0.70^{\mathrm{b}}$ & $7.26 \pm 0.76^{\mathrm{ab}}$ \\
50 & $8.79 \pm 0.98^{\mathrm{a}}$ & $12.27 \pm 0.75^{\mathrm{b}}$ & $10.16 \pm 0.81^{\mathrm{ab}}$ \\
25 & $12.33 \pm 0.80^{\mathrm{a}}$ & $15.41 \pm 0.67^{\mathrm{b}}$ & $12.93 \pm 0.73^{\mathrm{a}}$ \\
\hline
\end{tabular}

$a-b$ Letter superscripts are indicating significant differences

$(p \leq 0.05)$ between rows.

percentiles $(75,50$, and 25$)$. Focusing on the 50th percentile, $50 \%$ of the population fed with untreated Spirulina [E_S] survive until day 12 , meanwhile, in the control substrate [E_0], nematodes remain alive only until day 9, approximately. These results confirm what was previously mentioned for the cumulative survival curves. The lifespan of nematodes fed with a supplement of CP-treated Spirulina is in between the control and group fed with the supplement of non-treated Spirulina, specifically, values are higher than those reported for [E_0] but lower than the ones reported for [E_S]; however, values showed non-significant differences $(p>0.05)$ between either of the two. These findings are, probably, in concordance with previous results showing that the CP treatment applied was able to inactivate at least $2 \log 10$ of B. subtilis spores embedded in the Spirulina powder but, at the same time, a decrease in nutritive value, measured as a reduction in total phenolic compounds (TPC) and antioxidant activity (TEAC), was found for Spirulina CP treated samples (Beyrer et al., 2020).

The parameters of the fitted Weibull function for observed survivors (Figure 1) are given in Table 3. The group of nematodes fed with a Spirulina supplemented NGM [E_S] present higher value of $\partial$ (time to failure) (17.24 \pm 0.21 vs. $15.14 \pm 0.28$ for spirulina supplemented and control sample, respectively), which means that the death rate appears to be lower than that of the population fed in the absence of Spirulina. The kinetic constant $\widetilde{\partial}$ of the group fed with CP-treated Spirulina supplemented NGM () is, again, in between the two other groups but not significantly different from the one or the other $(15.14 \pm 0.28$, $17.24 \pm 0.21$, and $16.96 \pm 1.16$ for control, Spirulina and CPtreated Spirulina, respectively). So, the added nutritional value of CP-treated Spirulina CP cannot be confirmed statistically, which is in line with predictions with the Kaplan-Meier estimator. Consequently, the results showed that CP-treatment do not influence negatively the lifespan of $C$. elegans but a reduction in the Spirulina antiaging potential was found.

The C. elegans egg laying pattern is considered another valuable indicator of the in vivo impact associated to natural bioactivity, toxin exposure, and evaluation of other chemicalmediated disorders (Nidheesh et al., 2016; Teshiba et al., 2016; Peixoto et al., 2017; Salgueiro et al., 2017). Figure 2 shows the total number of eggs laid by each worm throughout its fertile phase. Nominally, the worms fed on the control substrate [E_0] laid more total eggs per individual than those fed on the substrate supplemented with CP-treated [E_SCP], or untreated [E_S] Spirulina powder. Statistically the differences were nonsignificant and a negative effect of Spirulina powder, CP treated or not, on egg laying of $C$. elegans cannot be concluded.

\section{Effect of Cold Atmospheric Pressure Plasma on the Antimicrobial Activity of Spirulina Assessed on Salmonella Typhimurium Infected Worms}

Caenorhabditis elegans has been used very frequently as a model of pathogenesis, and in particular for infection by pathogenic microorganisms (Balla and Troemel, 2013; Curt et al., 2014). In this sense, there are published studies in which the antimicrobial activity of a compound has been determined based on a higher survival of infected $C$. elegans when exposed to the antimicrobial compounds when compared with infected control nematodes (non-exposed to the antimicrobial compound) (SanzPuig et al., 2017; Ibáñez-Peinado et al., 2020; Palacios-Gorba et al., 2020). In the present study, an independent set of experiments was specifically designed to study the Spirulina (treated and untreated by CP) antimicrobial activity against Salmonella enterica serovar Typhimurium when C. elegans worms were infected.

Figure 3 shows the survival curves for the nematodes infected with $S$. Typhimurium and grown on a non-supplemented substrate [S_0] that will be used as a control, and on a substrate supplemented with untreated [S_S] and CP-treated Spirulina [S_SCP]. No significant differences on survival (neither changes in lifespan) $(p>0.05)$ of either non-treated or treated Spirulina with control sample was observed. Consequently, no reduction in infection was detected when infected nematodes were exposed to Spirulina, either untreated or treated by cold plasma.

In the same sense, data mining with the Kaplan-Meier estimation for computing the lifespan (Table 4) and fit parameters in the Weibull model for simulating the survival curve (Table 5), do not indicate differences between control samples [S_0] (nematodes fed without Spirulina supplementation) nor between nematodes with Spirulina supplementation when considering the impact of the plasma treatment [S_S] and [S_SCP]. The lifespan for percentiles $(75,50$, and $25 \%)$ of the population and kinetic constants $\partial$ in the Weibull model do not differ significantly for different substrates. Therefore, it can be concluded that the presence of Spirulina, treated or not by CAP, does not modify the survival of C. elegans, therefore, Spirulina does not present antimicrobial capacity in vivo against $S$. Typhimurium if it is determined by a reduced pathogenicity.

\section{DISCUSSION}

Spirulina has been incorporated as an ingredient of many recently launched food products (2015-2020: dairy productscheese, yogurt, smoothies; bakery products-cookies, bread, snacks; pasta; sauces, among others). This whole ingredient and its purified bioactive compounds are mostly commercialized 
TABLE 3 | Fit parameters of the survival curves (Weibull distribution function) for non-infected C. elegans populations fed with different substrates.

\begin{tabular}{|c|c|c|c|c|}
\hline & Kinetic constant $-\partial$ [worms/d] & Shape parameter $p[-]$ & $\mathbf{R}^{2}$ adjusted [-] & RMSE [-] \\
\hline Control [E_O] & $15.14 \pm 0.28^{a}$ & $1.96 \pm 0.25$ & 0.98 & 0.034 \\
\hline Spirulina [E_S] & $17.24 \pm 0.21^{b}$ & $2.73 \pm 0.19$ & 0.99 & 0.058 \\
\hline Spirulina CP-treated [E_SCP] & $16.96 \pm 1.16^{a b}$ & $2.12 \pm 0.36$ & 0.91 & 0.046 \\
\hline
\end{tabular}

${ }^{a-b}$ Different subscripts in the column indicate significant differences for $\mathrm{\partial}$.

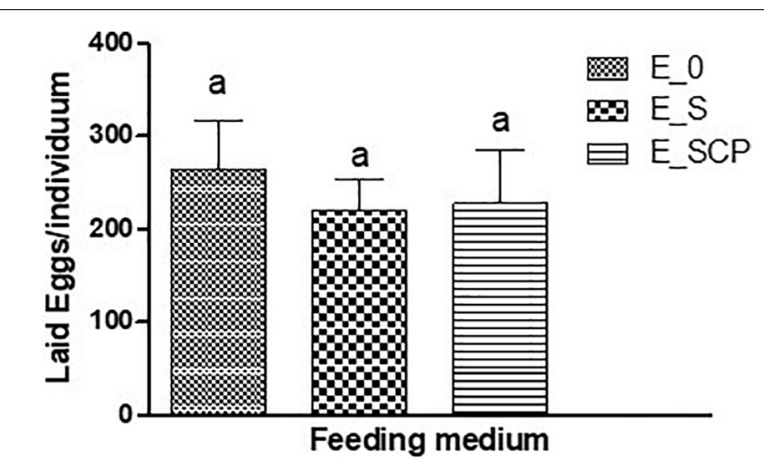

FIGURE 2 | Effect of feeding substrate on average egg laying per nematode of E. elegans: NGM without a supplement (control sample) [E_0]; NGM supplemented with non-treated Spirulina [E_S]; and NGM supplemented with CP-treated Spirulina [E_SCP].

and employed in powder form, to make up the final product, not only in the food industry but also in the pharmaceutical sector (development of novel nutraceutical products). These innovative matrices are subjected to different conventional and novel processing technologies (drying, lyophilization, roasting, thermal sterilization, extrusion, homogenization, fermentation) (Caporgno and Mathys, 2018), which can affect some macromolecules in the Spirulina processed matrix. Proteins and lipids in foods are prone to oxidation during industrial processing or storage; essential nutrients may be broken down and potentially toxic compounds might be generated (e.g., formation of 5-hydroxymethylfurfural and reduction of antioxidant activity after thermal treatment) (Kowalski, 2013; Winkler-Moser et al., 2020). Despite the positive antioxidant activity associated to Spirulina microalgae reported in the scientific literature, very few studies have evaluated the impact of novel processing technologies on this highly nutritious matrix (Colla et al., 2017; Beyrer et al., 2020). Indeed, it is important to gain greater insight into how these novel processes can degrade or affect the most unstable and valuable compounds (lipids, phycocyanin, polysaccharides, peptides, or vitamins) of Spirulina (Agustini et al., 2015; Caporgno and Mathys, 2018; Beyrer et al., 2020).

As for cold plasma (CP) as an innovative processing nonthermal technology, very few studies to date have reflected the physical-chemical analysis of CP processed food matrices, explaining the impact of complex plasma chemistry on food components (Bußler et al., 2016; Pankaj et al., 2018). Degradation of polysaccharides, loss of color, vitamin content, and total phenolic compounds, jointly with lipid oxidation have scarcely

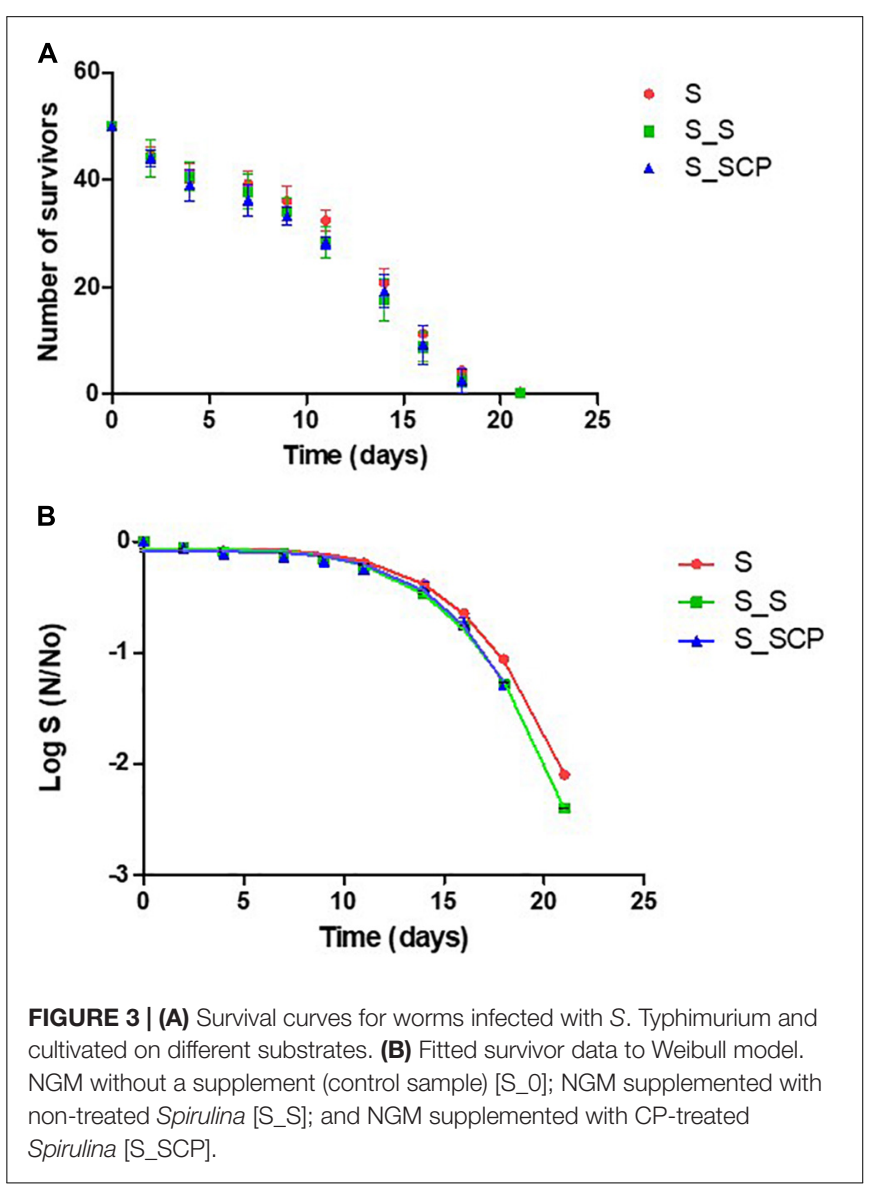

been described in vitro, with strong dependence on the type of plasma applied, gas carrier, and treatment intensity used (Grzegorzewski et al., 2011; Kim et al., 2013; Rodríguez et al., 2017). Han et al. (2016) studied the in vivo effect of CP food processed matrices assessing possible associated risks. Results revealed no acute toxicity associated to $\mathrm{CP}$-treated soy-based edible films assessed in a rat model (Han et al., 2016). However, to date there are hardly any studies published on cold plasma effects of food bioactivity in vivo.

According to our results, it seems that no-negative effect is derived from SMD-CAPP treatment of Spirulina (3.3 W power discharge; $7 \mathrm{~min}$ ) that could diminish the survival of C. elegans worms; however, it cannot be concluded the same bioactive/antiaging properties of the processed product as shown by in vivo assays in the C. elegans model. Previous studies have demonstrated the potential of $C$. elegans to test antioxidant/anti-aging in vivo properties associated to other 
TABLE 4 | Lifespan (days) of infected nematodes for the different percentile depending on group studied.

\begin{tabular}{lccc}
\hline $\begin{array}{l}\text { Percentile of } \\
\text { survivors [\%] }\end{array}$ & \multicolumn{3}{c}{ Lifespan of nematodes [d] } \\
\cline { 2 - 4 } & Control [S_0] & $\begin{array}{c}\text { Untreated } \\
\text { Spirulina } \\
\text { [S_S] }\end{array}$ & $\begin{array}{c}\text { CP treated } \\
\text { Spirulina } \\
\text { [S_SCP] }\end{array}$ \\
\hline 75 & $8.53 \pm 1.25^{\mathrm{a}}$ & $6.99 \pm 1.36^{\mathrm{a}}$ & $5.88 \pm 1.98^{\mathrm{a}}$ \\
50 & $13.07 \pm 0.39^{\mathrm{a}}$ & $12.07 \pm 0.79^{\mathrm{a}}$ & $12.25 \pm 0.47^{\mathrm{a}}$ \\
25 & $15.9 \pm 0.35^{\mathrm{a}}$ & $15.20 \pm 0.64^{\mathrm{a}}$ & $15.47 \pm 0.67^{\mathrm{a}}$
\end{tabular}

a Letter superscript indicate significant differences $(p \leq 0.05)$ between rows.

natural compounds, such as green tea, purple wheat or açai (Euterpe precatoria Mart.) (Chen et al., 2013; Abbas and Wink, 2014; Peixoto et al., 2016). This animal model was also used by Wilson et al. (2010) to evaluate the bioactivity of blueberry proanthocyanins and a collection of resveratrol analogs, concluding that these phytochemicals enhance longevity and stress resistance of $C$. elegans adult worms. More recently, Ibáñez-Peinado et al. (2020) studied the effect of a cauliflower extract on nematode lifespan in a population fed on enriched media. Results indicated that the cauliflower extract had a protective effect on nematodes senescence. Authors reported a percentile 50 of 12.4 days, representing a significant increase in comparison with the 6.92 days for nematodes lifespan when fed on the substrate not supplemented with cauliflower extract (NMG). The results obtained by Ibáñez-Peinado et al. (2020) are similar to those obtained in the present work for the same percentile 50th, with C. elegans lifespan being increased significantly when fed on Spirulina (untreated) supplemented substrates (12.27 vs. 8.79 days in NMG non supplemented). As indicated by Ibáñez-Peinado et al. (2020) and also by Fang et al. (2019), it is likely that natural extracts from plants and, in this case from algae, would produce an antioxidant effect by means the up-regulating the expression of antioxidant-related genes in C. elegans, and inhibiting cell apoptosis, improving the nematode antioxidant defense system, leading to the lengthening of the lifespan.

With respect to microalgae antioxidants, Beyrer et al. (2020) demonstrated that no significant differences $(p>0.05)$ were detected on total phenolic content (TPC) of treated Spirulina powder (SMD-CAPP ignited on air range 1.1-2.2 $\mathrm{W}$ discharge power), however, the antioxidant potential attributed to Spirulina powder was slightly reduced when exposed to that specific treatment. This reduction seems to be related with the slight reduction in the in vivo antiaging potential of Spirulina described in the present manuscript, although non-significant differences have been detected between nematodes lifespan when fed with untreated vs. CAPP treated Spirulina, even under CAPP ignited on air-3.3 W treatments, applied at longer treatment times (7 $\mathrm{min})$.

In relation to the antimicrobial bioactive potential of Spirulina, it has been extensively reported in vitro (AlGhanayem, 2017; Pina-Pérez et al., 2018). To date, C. elegans has been used as in vivo model for novel antimicrobial drug research, to study therapies against Staphylococcus aureus, Pseudomonas aeruginosa, Enterococcus faecalis, and H. pylori (Kong et al., 2016). The present results are the first to provide an in vivo approach to evaluate the antimicrobial potential of Spirulina powder $(1 \mathrm{mg} / \mathrm{mL})$ against Salmonella enterica serovar Typhimurium. However, no significant antimicrobial activity was detected in vivo due to $C$. elegans exposure to the Spirulina concentration assayed $(1 \mathrm{mg} / \mathrm{mL})$. Neither did the air ignited cold plasma provided any positive or negative antimicrobial effect on infected $C$. elegans worms, with similar lifespan rates as those in control samples without Spirulina. Other algae compounds have demonstrated antimicrobial potential in vivo using the C. elegans animal model (PalaciosGorba et al., 2020). According to these authors, fucoidan (a sulphated polysaccharide from Phaeophyceae) demonstrates potential antimicrobial activity in vivo against Helicobacter pylori, when administered in the range 50-200 $\mu \mathrm{g} / \mathrm{ml}$. Also, Ibáñez-Peinado et al. (2020) demonstrated that a cauliflower extract increased the survival of a $C$. elegans population infected with Salmonella enterica, obtaining 50th percentile values equivalent to 8.8 days in supplemented substrate (NMG+cauliflower), in relation to 50th percentile of 4.4 days for a nematode population infected and fed on NMG plates (not supplemented).

The present study is the first to provide mathematical modeling of C. elegans survival under Spirulina exposure (CPtreated and untreated), via use of the Weibull distribution function. The value of $\mathrm{p}$ (shape parameter) has a marked effect on the failure rate (worm death rate) of the Weibull distribution. Inferences can be drawn about a population's failure characteristics (worm death rate) by considering whether the value of $\mathrm{p}$ is less than, equal to, or greater than one. If $p<1$, the model exhibits a failure rate that decreases with time, in populations where $p=1$ there is a constant failure rate (consistent with the exponential distribution), and populations with $p>1$ have a failure rate that increases with time, as in the case of this study. Probably this behavior is linked to the age of worms and reflects the senescence process. At the same time, the Weibull distribution function provides kinetic parameters to objectively describe survival curves of exposed nematodes, either uninfected

TABLE 5 | Fit parameters of the survival curves (Weibull distribution function) for infected C. elegans populations fed with different substrates.

\begin{tabular}{|c|c|c|c|c|}
\hline & Kinetic constant $\partial$ [worms/d] & Shape parameter $p[-]$ & $\mathbf{R}^{2}$ adjusted [-] & RMSE [-] \\
\hline Control [S_0] & $18.00 \pm 0.31^{a}$ & $4.58 \pm 0.16$ & 0.99 & 0.032 \\
\hline Spirulina [S_S] & $17.28 \pm 0.23^{a}$ & $4.34 \pm 0.16$ & 0.99 & 0.039 \\
\hline Spirulina CP-treated [S_SCP] & $17.38 \pm 0.37^{\mathrm{a}}$ & $4.75 \pm 0.53$ & 0.98 & 0.053 \\
\hline
\end{tabular}

a Different subscripts in the column indicate significant differences for $\mathrm{\partial}$. 
or infected by Salmonella and exposed to CP-treated Spirulina, and quantify worm death rate under different study conditions.

\section{CONCLUSION}

Surface Microdischarge Cold Atmospheric Pressure Plasma is currently considered an effective new technology to sterilize food and pharmaceutical matrices, in a few minutes ( $<7 \mathrm{~min})$, when plasma is ignited on air (cost-effective process). However, further research is required in relation to food macromolecules stability/functionality under such new treatments, mainly considering the dynamic chemistry generated during foodplasma interaction. According to the results of the present study, no negative effects were recorded on C. elegans indicators, such as lifespan and reproductive rate after SMD-CAPP treatment (no reduction was detected). Validation of Spirulina bioactivity (i.e., improved C. elegans lifespan) has been demonstrated and mathematically modeled for the first time. No antimicrobial effect, measured as an increase in C. elegans lifespan, was detected in worms infected with Salmonella enterica serovar Typhimurium when Spirulina was added to the media a concentration of $1 \mathrm{mg} / \mathrm{ml}$.

\section{REFERENCES}

Abbas, S., and Wink, M. (2014). Green tea extract induces the resistance of Caenorhabditis elegans against oxidative stress. Antioxidants 3, 129-143. doi: 10.3390/antiox3010129

Agustini, T. W., Suzery, M., and Sutrisnanto, D. (2015). Comparative study of bioactive substances extracted from fresh and dried Spirulina sp. Procedia Environ. Sci. 23, 282-289. doi: 10.1016/j.proenv.2015.01.042

Al-Ghanayem, A. A. (2017). Antimicrobial activity of Spirulina platensis extracts against certain pathogenic bacteria and fungi. $A d v$. Biores. 8, 96-101. doi: 10. 15515/abr.0976-4585.8.6.96101

Alves Filho, E. G., Silva, L. M. A., Filho, F. O., Rodrigues, S., Fernandes, F. A. N., Gallão, M. I., et al. (2019). Cold plasma processing effect on cashew nuts composition and allergenicity. Food Res. Int. 125, 1-9. doi: 10.1016/j.foodres. 2019.108621

Ayyadevara, S., Bharill, P., Dandapat, A., Hu, C., Khaidakov, M., Mitra, S., et al. (2013). Aspirin inhibits oxidant stress, reduces age-associated functional declines, and extends lifespan of Caenorhabditis elegans. Antiox. Redox Signal. 18, 481-490. doi: 10.1089/ars.2011.4151

Balla, K. M., and Troemel, E. R. (2013). Caenorhabditis elegans as a model for intracellular pathogen infection. Cell. Microbiol. 15, 1313-1322. doi: 10.1111/ cmi. 12152

Beyrer, M., Pina-Perez, M. C., Martinet, D., and Andlauer, W. (2020). Cold plasma processing of powdered Spirulina algae for spore inactivation and preservation of bioactive compounds. Food Control 118, 1-8. doi: 10.1016/j.foodcont.2020. 107378

Bußler, S., Rumpold, B. A., Fröhling, A., Jander, E., Rawel, H. M., and Schlüter, O. K. (2016). Cold atmospheric pressure plasma processing of insect flour from Tenebrio molitor: impact on microbial load and quality attributes in comparison to dry heat treatment. Innov. Food Sci. Emerg. Technol. 36, 277-286. doi: 10.1016/j.ifset.2016.07.002

Caporgno, M. P., and Mathys, A. (2018). Trends in microalgae incorporation into innovative food products with potential health benefits. Front. Nutr. 5:58. doi: 10.3389/fnut.2018.00058

Cassani, L., Gomez-Zavaglia, A., Jimenez-López, C., Lourenço-Lopes, C., Prieto, M. A., and Simal-Gandara, J. (2020). Seaweed-based natural ingredients: stability of phlorotannins during extraction, storage, passage through the

\section{DATA AVAILABILITY STATEMENT}

The raw data supporting the conclusions of this article will be made available by the authors, without undue reservation.

\section{AUTHOR CONTRIBUTIONS}

DR and AM: conceptualization. DR, MÚ-M, and MP-P: methodology. MÚ-M and MP-P: experimental work. MP-P, AM, $\mathrm{DR}$, and $\mathrm{MB}$ : writing-original draft preparation, writingreview and editing, and funding acquisition. All authors have read and agreed to the published version of the manuscript.

\section{FUNDING}

This work was supported by funds provided by the Spanish Ministry of Science, Innovation and Universities and FEDER funds (AGL017-86840-C2-2-R and PID2020-116318RB-C31). The European Commission funded this research in the frame of the Marie Curie Skłodowska Action (H2020 MCSAIF EU 748314).

gastrointestinal tract and potential incorporation into functional foods. Food Res. Int. 137, 1-14. doi: 10.1016/j.foodres.2020.109676

Chaiklahan, R., Chirasuwan, N., and Bunnag, B. (2012). Stability of phycocyanin extracted from Spirulina sp.: influence of temperature, $\mathrm{pH}$ and preservatives. Process. Biochem. 47, 659-664. doi: 10.1016/j.procbio.2012. 01.010

Chen, W., Müller, D., Richling, E., and Wink, M. (2013). Anthocyanin-rich purple wheat prolongs the life span of Caenorhabditis elegans probably by activating the daf-16/foxo transcription factor. J. Agric. Food Chem. 61, 3047-3053. doi: 10.1021/jf3054643

Chen, Y., Wan, X., Wu, D., Ouyang, Y., Gao, L., Chen, Z., et al. (2020). Characterization of the structure and analysis of the anti-oxidant effect of microalga Spirulina platensis polysaccharide on Caenorhabditis elegans mediated by modulating microRNAs and gut microbiota. Int. J. Biol. Macromol. 163, 2295-2305. doi: 10.1016/j.ijbiomac.2020.09.041

Colla, L. M., Bertol, C. D., Ferreira, D. J., Bavaresco, J., Costa, J. A. V., and Bertolin, T. E. (2017). Thermal and photo-stability of the antioxidant potential of Spirulina platensis powder. Braz. J. Biol. 77, 332-339. doi: 10.1590/1519-6984. 14315

Curt, A., Zhang, A., Minnerly, J., and Jia, K. (2014). Intestinal autophagy activity is essential for host defense against Salmonella typhimurium infection in Caenorhabditis elegans. Dev. Comp. Immunol. 45, 214-218. doi: 10.1016/j.dci. 2014.03.009

El-Baky, H. H. A., El Baz, F. K., and El-Baroty, G. S. (2008). Characterization of nutraceutical compounds in blue green alga Spirulina maxima. J. Med. Plants Res. 2, 292-300. doi: 10.5897/JMPR.9000331

Elmoualij, B., Thellin, O., Gofflot, S., Heinen, E., Levif, P., Séguin, J., et al. (2012). Decontamination of prions by the flowing afterglow of a reduced-pressure $\mathrm{N}_{2}-\mathrm{O}_{2}$ cold-plasma. Plasma Proc. Polym. 9, 612-618. doi: 10.1002/ppap.20110 0194

Fang, Z., Chen, Y., Wang, G., Feng, T., Shen, M., Xiao, B., et al. (2019). Evaluation of the antioxidant effects of acid hydrolysates from Auricularia auricular polysaccharides using a Caenorhabditis elegans model. Food Funct. 10, 55315543. doi: 10.1039/C8FO02589D

Gavahian, M., Chu, Y. H., Khaneghah, A. M., Barba, F. J., and Misra, N. N. (2018). A critical analysis of the cold plasma induced lipid oxidation in foods. Trends Food Sci. Technol. 77, 32-41. doi: 10.1016/j.tifs.2018.04.009 
Geeraerd, A. H., Valdramidis, V. P., and Van Impe, J. F. (2005). GInaFiT, a freeware tool to assess non-log-linear microbial survivor curves. Int. J. Food Microbiol. 102, 95-105. doi: 10.1016/j.ijfoodmicro.2004.11.038

Grahl, S., Strack, M., Weinrich, R., and Mörlein, D. (2018). Consumer-Oriented product development: the conceptualization of novel food products based on Spirulina (Arthospira platensis) and resulting consumer expectations. J. Food Qual. 2018:1919482. doi: 10.1155/2018/1919482

Grzegorzewski, F., Ehlbeck, J., Schlüter, O., Kroh, L. W., and Rohn, S. (2011). Treating lamb's lettuce with a cold plasma-influence of atmospheric pressure air plasma immanent species on the phenolic profile of Valerianella locusta. LWT Food Sci. Technol. 44, 2285-2289. doi: 10.1016/j.lwt.2011.05.004

Han, S. H., Suh, H. J., Hong, K. B., Kim, S. Y., and Min, S. C. (2016). Oral toxicity of cold plasma-treated edible films for food coating. J. Food Sci. 81, 3052-3057. doi: 10.1111/1750-3841.13551

Ibáñez-Peinado, D., Pina-Pérez, C., García-Carrión, G., Martínez, A., and Rodrigo, D. (2020). In vivo antimicrobial activity assessment of a cauliflower by-product extract against Salmonella typhimurium. Front. Sustain. Food Syst. 4:8. doi: $10.3389 /$ fsufs.2020.00008

Jattujan, P., Chalorak, P., Siangcham, T., Sangpairoj, K., Nobsathian, S., Poomtong, T., et al. (2018). Holothuria scabra extracts possess anti-oxidant activity and promote stress resistance and lifespan extension in Caenorhabditis elegans. Exp. Gerontol. 110, 158-171. doi: 10.1016/j.exger.2018.06.006

Kaplan, E. L., and Meier, P. (1958). Nonparametric estimation from incomplete observations. JASA 53, 457-481.

Khan, M., Shobha, C. J., Mohan, J. I., Rao, U. M., Prayag, A. N., and Kutala, K. V. (2006). Spirulina attenuates cyclosporine-induced nephrotoxicity in rats. J. Appl. Toxicol. 26, 444-451. doi: 10.1002/jat.1159

Khan, M., Shobha, C. J., Rao, U. M., Sundaram, C. M., Singh, S., Mohan, J. I., et al. (2005). Protective effect of Spirulina against doxorubicin-induced cardiotoxicity. Phytother. Res. 19, 1030-1037. doi: 10.1002/ptr.1783

Kim, H.-J., Yong, H. I., Park, S., Choe, W., and Jo, C. (2013). Effects of dielectric barrier discharge plasma on pathogen inactivation and the physicochemical and sensory characteristics of pork loin. Curr. Appl. Phys. 13, 1420-1425. doi: 10.1016/j.cap.2013.04.021

Kong, C., Eng, S. A., Lim, M. P., and Nathan, S. (2016). Beyond traditional antimicrobials: a Caenorhabditis elegans model for discovery of novel antiinfectives. Front. Microbiol. 7:1956. doi: 10.3389/fmicb.2016.01956

Kowalski, S. (2013). Changes of antioxidant activity and formation of 5hydroxymethylfurfural in honey during thermal and microwave processing. Food Chem. 15, 1378-1382. doi: 10.1016/j.foodchem.2013.04.025

Lee, S.-Y., Kim, K.-B.-W.-R., Lim, S., and Ahn, D.-H. (2014). Antibacterial mechanism of Myagropsis myagroides extract on Listeria monocytogenes. Food Control 42, 23-28. doi: 10.1016/j.foodcont.2014.01.030

Mafart, P., Couvert, O., Gaillard, S., and Leguerinel, I. (2002). On calculating sterility in thermal preservation methods: application of the Weibull frequency distribution model. Int. J. Food Microbiol. 72, 107-113. doi: 10.1016/S01681605(01)00624-9

Marco Castro, E., Shannon, E., and Abu-Ghannam, N. (2019). Effect of fermentation on enhancing the nutraceutical properties of Arthospira platensis (Spirulina). Ferment. 5, 1-19. doi: 10.3390/fermentation5010028

Martelli, F., Cirlini, M., Lazzi, C., Neviani, E., and Bernini, V. (2020). Edible seaweeds and spirulina extracts for food application: in vitro and in situ evaluation of antimicrobial activity towards foodborne pathogenic bacteria. Foods 9, 1-15. doi: 10.3390/foods9101442

Nidheesh, T., Salim, C., Rajini, P. S., and Suresh, P. V. (2016). Antioxidant and neuroprotective potential of chitooligomers in Caenorhabditis elegans exposed to monocrotophos. Carbohydr. Polym. 135, 138-144. doi: 10.1016/j.carbpol. 2015.08.055

Nuhu, A. A. (2013). Spirulina (Arthospira): an important source of nutritional and medicinal compounds. J. Mar. Sci. 2013:325636. doi: 10.1155/2013/325636

Ozdemir, G., Karabay, N. U., Dalay, C. M., and Pazarbasi, B. (2004). Antibacterial activity of volatile component and various extracts of Spirulina platensis. Phytoth. Res. 18, 754-757. doi: 10.1002/ptr.1541

Palacios-Gorba, C., Pina, R., Tortajada-Girbés, M., Jimenez-Belenguer, A., Siguemoto, E., Ferrús, M. A., et al. (2020). Caenorhabditis elegans as an in vivo model to assess fucoidan bioactivity preventing Helicobacter pylori infection. Food Funct. 11, 4525-4534. doi: 10.1039/D0FO00768D
Pankaj, S. K., Wan, Z., and Keener, K. M. (2018). Effects of cold plasma on food quality: a review. Foods 7:4. doi: 10.3390/foods7010004

Peixoto, H., Roxo, M., Krstin, S., Röhrig, T., Richling, E., and Wink, M. (2016). An anthocyanin-rich extract of acai (Euterpe precatoria Mart.) increases stress resistance and retards aging-related markers in Caenorhabditis elegans. J. Agric. Food Chem. 64, 1283-1290. doi: 10.1021/acs.jafc.5b 05812

Peixoto, H., Roxo, M., Röhring, T., Riching, E., Wang, X., and Wink, M. (2017). Anti-aging and antioxidant potential of Paullinia cupana var. sorbilis: findings in Caenorhabditis elegans indicate a new utilization for roasted seeds of guarana. Medicines 4:61. doi: 10.3390/medicines4030061

Pérez-Andrés, J. M., Álvarez, C., Cullen, P. J., and Tiwari, B. K. (2019). Effect of cold plasma on the techno-functional properties of animal protein food ingredients. Innov. Food Sci. Emerg. Technol. 58, 1-7. doi: 10.1016/j.ifset.2019.10 2205

Pina-Pérez, M. C., Martinet, D., Palacios-Gorba, C., Ellert, C., and Beyrer, M. (2020). Low-energy short-term cold atmospheric plasma: controlling the inactivation efficacy of bacterial spores in powders. Food Res. Int. 130, 1-10. doi: 10.1016/j.foodres.2019.108921

Pina-Pérez, M. C., Rivas, A., Martínez, A., and Rodrigo, D. (2017). Antimicrobial potential of macro and microalgae against pathogenic and spoilage microorganisms in food. Food Chem. 235, 34-44. doi: 10.1016/j.foodchem.2017.05.033

Pina-Pérez, M. C., Rivas, A., Martinez, A., and Rodrigo, D. (2018). Effect of thermal treatment, microwave, and pulsed electric field processing on the antimicrobial potential of açaí (Euterpe oleracea), stevia (Stevia rebaudiana Bertoni), and ginseng (Panax quinquefolius L.) extracts. Food Control 90, 98-104. doi: 10. 1016/j.foodcont.2018.02.022

Rodríguez, Ó, Gomes, W. F., Rodrigues, S., and Fernandes, F. A. (2017). Effect of indirect cold plasma treatment on cashew apple juice (Anacardium occidentale L.). LWT Food Sci. Technol. 84, 457-463.

Salgueiro, W. G., Goldani, B. S., Peres, T. V., Miranda-Vizuete, A., Aschner, M., Teixeirada Rocha, J. B., et al. (2017). Insights into the differential toxicological and antioxidant effects of 4-phenylchalcogenil-7-chloroquinolines in Caenorhabditis elegans. Free Radic. Biol. Med. 110, 133-141. doi: 10.1016/j. freeradbiomed.2017.05.020

Sanz-Puig, M., Arana-Lozano, A., Pina-Pérez, M. C., Fernandez, P., Martinez, A., and Rodrigo, D. (2020). Occurrence of Salmonella typhimurium resistance under sublethal/repeated exposure to cauliflower infusion and infection effects on Caernohabditis elegans host test organism. Food Sci. Technol. Int. 26, 151159. doi: 10.1177/1082013219873500

Sanz-Puig, M., Lázaro, E., Armero, C., Alvares, D., Martínez, A., and Rodrigo, D. (2017). S. typhimurium virulence changes caused by exposure to different non-thermal preservation treatments using C. elegans. Int. J. Food Microbiol. 4, 49-54. doi: 10.1016/j.ijfoodmicro.2017.09.006

Sarangapani, C., Keogh, D. R., Dunne, J., Bourke, P., and Cullen, P. J. (2017). Characterisation of cold plasma treated beef and dairy lipids using spectroscopic and chromatographic methods. Food Chem. 235, 324-333. doi: 10.1016/j.foodchem.2017.05.016

Seghiri, R., Kharbach, M., and Essamri, A. (2019). Functional composition, nutritional properties and biological activities of Moroccan Spirulina microalga. J. Food Quality 2019, 1-12. doi: 10.1155/2019/3707219

Stiernagle, T. (2006). Maintenance of C. elegans The C. elegans Research Community, WormBook (eds). 1-11. Available online at: http://www. wormbook.org/chapters/www_strainmaintain/strainmaintain.html (accessed January 10, 2022).

ten Bosch, L., Pfohl, K., Avramidis, G., Wieneke, S., Viöl, W., and Karlovsky, P. (2017). Plasma-based degradation of mycotoxins produced by Fusarium, Aspergillus and Alternaria species. Toxins (Basel) 9:97. doi: 10.3390/ toxins 9030097

Teshiba, E., Miyahara, K., and Takeya, H. (2016). Glucose-induced abnormal egg-laying rate in Caenorhabditis elegans. Biosci. Biotechnol. Biochem. 80, 14361439. doi: 10.1080/09168451.2016.1158634

Tolouie, H., Mohammadifar, M. A., Ghomi, H., and Hashemi, M. (2018). Cold atmospheric plasma manipulation of proteins in food systems. Crit. Rev. Food Sci. Nutr. 58, 2583-2597. doi: 10.1080/10408398.2017.133 5689 
Whitehead, J. C. (2016). "Chapter 3- The chemistry of cold plasma," in Cold Plasma in Food and Agriculture Fundamentals and Applications, eds N. N. Misra, O. Schlüter, and P. J. Cullen (Amsterdam: Academic Press), 53-81. doi: 10.1016/B978-0-12-801365-6.00 003-2

Wilson, M. A., Hunt, P. R., and Wolkow, C. A. (2010). "Using Caenorhabditis elegans to study bioactivities of natural products from small fruits," in Flavor and Health Benefits of Small Fruits, eds M. C. Qian and A. M. Rimando (Washington, DC: ACS Symposium Series), 227-238. doi: 10.1021/bk-20101035.ch014

Winkler-Moser, J. K., Hwang, H. S., and Kerr, B. J. (2020). Changes in markers of lipid oxidation and thermal treatment in feed-grade fats and oils. J. Sci. Food Agric. 100, 3328-3340. doi: 10.1002/jsfa.10364

Ziuzina, D., and Misra, N. N. (2016). "Chapter 9-Cold plasma for food safety," in Cold Plasma in Food and Agriculture Fundamentals and Applications, eds N. N. Misra, O. Schlüter, and P. J. Cullen (London: Academic Press), 223-252.
Conflict of Interest: The authors declare that the research was conducted in the absence of any commercial or financial relationships that could be construed as a potential conflict of interest.

Publisher's Note: All claims expressed in this article are solely those of the authors and do not necessarily represent those of their affiliated organizations, or those of the publisher, the editors and the reviewers. Any product that may be evaluated in this article, or claim that may be made by its manufacturer, is not guaranteed or endorsed by the publisher.

Copyright (c) 2022 Pina-Pérez, Úbeda-Manzanaro, Beyrer, Martínez and Rodrigo. This is an open-access article distributed under the terms of the Creative Commons Attribution License (CC BY). The use, distribution or reproduction in other forums is permitted, provided the original author $(s)$ and the copyright owner(s) are credited and that the original publication in this journal is cited, in accordance with accepted academic practice. No use, distribution or reproduction is permitted which does not comply with these terms. 